\title{
The Effect of Atmospheric Plasma on the Shipping Stability of Powdered Whey Protein Isolate
}

Michael D Birnbaum

Department of Molecular and Cellular Pharmacology, University of Miami, United States

Email: MBirnbaum@med.miami.edu 


\begin{abstract}
:
Whey protein powders are the most nutritionally available proteins on the market. As such, their accessibility has led to a wide commercialization including online retail, which in turn has resulted in these products being exposed to several shipping and handling conditions that could adversely affect their structural integrity and therefore their efficacy and nutritional value. The objective of this study was to investigate the ability of commercially available whey protein powder isolate to structurally withstand various shipping and handling conditions and whether that resilience could be enhanced via PSM treatment. Using Protein Thermal Shift Assay (PTSA), we demonstrated various structural changes in whey protein isolate powder as a result of shipping methods, whereby 2-3 day shipping had the least structural impact, followed by ground shipping. The highest structural impact was observed with the shortest transportation method, namely 1-day shipping, where a $9.14 \%$ change in structure was observed via PTSA. Furthermore, PSM treated whey powders had an overall $58.04 \%$ lower structural variation than their nontreated counterparts. Overall, we demonstrate that commercially available whey protein powder isolates undergo significant structural variations due to shipping and handling. We also show that PSM treatment lessens those variations and as such serves as an effective method in preserving protein structure of commercially available whey powder isolates.
\end{abstract}

Figures: 
A.

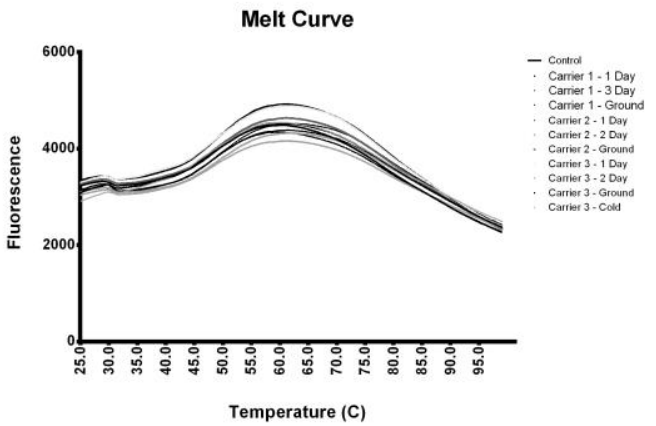

C.

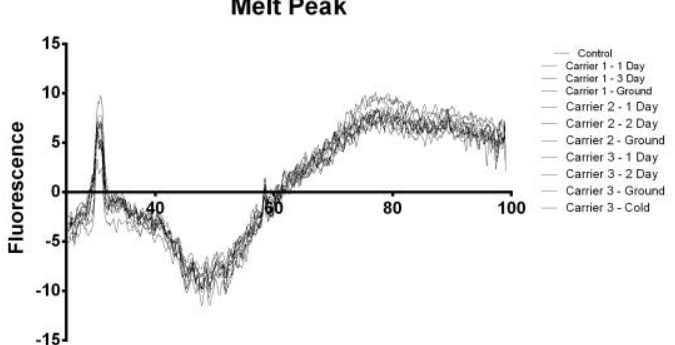

Temperature (C)

E

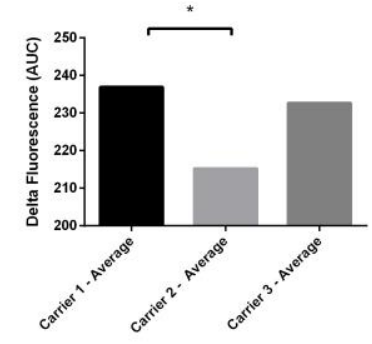

G.

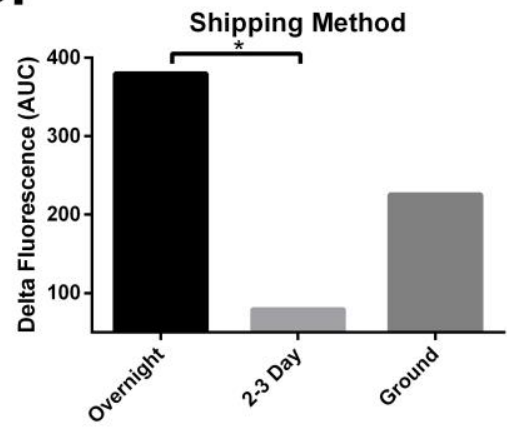

B.
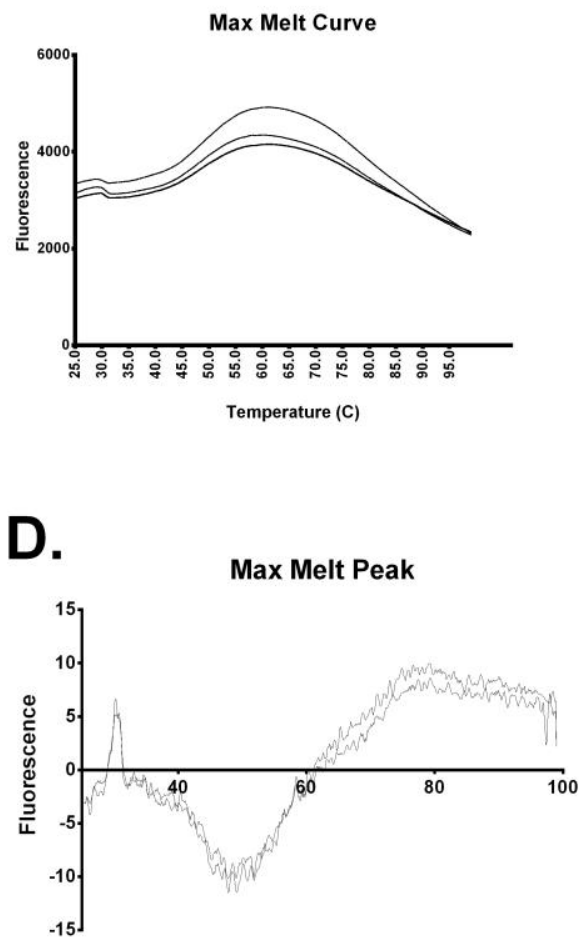

Temperature (C)

F. Cold Shipping Vs. Ambient Shipping

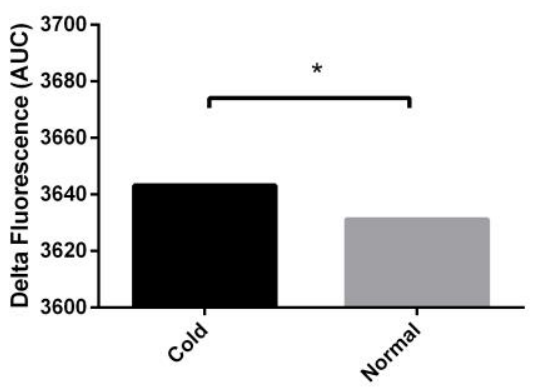

H.

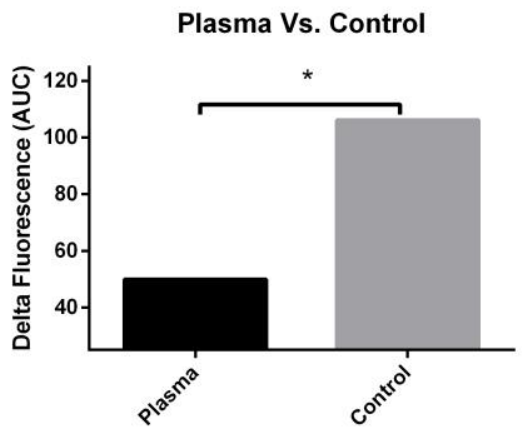




\section{Figure Legend:}

Figure A: Comparison of Melt Curve measurements of Carrier 1 - 1 Day, Carrier 1 - 3, Day, Carrier 1 - Ground, Carrier 2 - 1 Day, Carrier 2 - 2 Day, Carrier 2 - Ground, Carrier 3 - 1 Day, Carrier 3 - 2 Day, Carrier 3 - Ground, Carrier 3 - Cold sample as compared to untreated protein.

Figure B: Comparison of Melt Curve measurements of Carrier 1 - 1 day sample, PSM altered protein, as compared to untreated protein.

Figure C: Comparison of Melt Curve measurements of Carrier 1 - 1 Day, Carrier 1 - 3 Day, Carrier 1 - Ground, Carrier 2 - 1 Day, Carrier 2 - 2 Day, Carrier 2 - Ground, Carrier 3 - 1 Day, Carrier 3 - 2 Day, Carrier 3 - Ground, Carrier 3 - Cold sample as compared to untreated protein.

Figure D: Comparison of Melt Peak measurements of 1 day sample, PSM altered protein, as compared to untreated protein.

Figure E: Comparison of Melt Curve, Area Under Curve of Carrier 1 Average, Carrier 2 Average, Carrier 3 Average. Asterisk ( ${ }^{*}$ ) indicates $9.14 \%$ decrease in melt curve alteration by transportation.

Figure F: Comparison of Melt Curve, Area Under Curve of normal ambient shipment, compared to a thermally stable shipment $\left(2^{\circ} \mathrm{C}\right.$ to $8^{\circ} \mathrm{C}$ environment). Asterisk ( $\left.{ }^{*}\right)$ indicates $0.33 \%$ increase in melt curve alteration by transportation.

Figure G: Comparison of Melt Curve, Area Under Curve of overnight shipping, 2-3 day shipping and ground shipping. Asterisk $\left({ }^{*}\right)$ indicates $79.08 \%$ decrease in melt curve alteration by transportation.

Figure H: Comparison of Melt Curve, Area Under Curve of PSM altered protein and a control protein. Asterisk $\left(^{*}\right)$ indicates $53.02 \%$ decrease in melt curve alteration by transportation.

\section{Introduction:}

Proteins as isolates and concentrates are in high demand as they are essential constituents of many food products due to their variable characteristics and functions. ${ }^{8}$ As they possess various and dynamic structural and physiochemical properties, whey protein products are ideal food product ingredients, serving several functions such as flavor enhancers, preservatives and texture enhancers. ${ }^{8,9}$ Whey proteins have also been used as healthy nutritional alternatives, whereby they serve as a rich source of amino 
acids and as such provide an effective dietary method for weight management. ${ }^{9,10}$ Dietary whey protein has also been considered as an effective cancer-reducing treatment regimen. ${ }^{9}$ Given their various functions, it is essential that protein structures are preserved as any loss can lead to variations in functionality which can result in either decrease or loss of efficacy, all of which are undesirable outcomes that could manifest themselves as either loss in flavor or product stability. It is therefore of much interest to study and understand how protein structures change as a result of different circumstances so that measures could be design to minimize undesirable structural consequences.

Plasma-surface modification (PSM) is a process whereby plasma, once in contact with the surface of a material, causes alterations in its chemical properties, such as surface area and hydrophilicity. ${ }^{5}$ When an electrical field is applied to a gas under conditions of partial vacuum, a partially ionized gas is obtained. ${ }^{6}$ When a gas is introduced into a vacuum chamber, treated with an electromagnetic field and allowed to interact with another material, the surface structure of that material will be altered by the plasma. ${ }^{6}$ Atmospheric plasma is an effective means to cause structural changes of organic protein powders, ${ }^{7}$ and its function as a preserving or protective method of protein resilience to external conditions has not been studied yet. This study assesses PSM-induced protein alterations as a means to preserve protein structure changes that may be the results of shipping and handling procedures.

PSM-induced protein changes are measured via the Protein Thermal Shift Assay (PTSA) in this study. The PTSA has become a popular protein melt analysis method as an efficient and cost effective protein melt screening process. As it is based on very small amounts of starting material, it is more economical and material friendly. PTSA is based on gradual increases in the melting temperature that yields a 'thermal shift,' based on which stabilization of the protein under various conditions can be assessed. ${ }^{1}$ Protein folding and unfolding characteristics have been studied for several purposes, including drug target interaction efforts. ${ }^{2,3,4}$ Experimentally, Protein Thermal Shift dye binds to hydrophobic regions that are exposed as a result of the increase in temperature, and this results in a measurable fluoresce, whose outputs are melt curves or melt peaks of a specific protein as indicators of protein folding extent.

\section{Objective:}

The objective of this study is to investigate the thermal stability of dry PSM treated and non-treated powdered whey protein isolate under various shipping and transportation conditions using a protein thermal shift assay. 


\section{Results \& Discussion:}

Dry powdered whey protein isolate was altered using PSM in order to assess whether those structural changes result in increased resilience to shipping and handling conditions. Proprietary PSM technology (Ingredient Optimized TM, Plasma Nutrition, USA) was used to effectively mediate the exposure of organic dry powder materials to plasma to enact multiple changes, including protein structure alterations. A commercially available dry powder whey protein isolate was utilized as source material, which served both as a control as well as treated material. A Protein Thermal Shift assay (PTSA) was conducted on both pristine samples of whey protein isolate (control), samples of whey protein isolate exposed to various shipping conditions from the same batch (experimental) and samples of whey protein isolate exposed to atmospheric plasma and shipping conditions (experimental).

We report both protein melt curves (Figure $A$ ) as well as melt peaks (Figure $C$ ) for whey protein samples that were exposed to a total of 10 shipping and handling conditions across three different commercially available carriers. The protein melt curves and melt peaks that that indicate largest difference in structural alterations are illustrated in Figures $B$ and $D$, respectively. Furthermore, when the shipping-induced protein alterations were compared, average PTSA fluorescence values demonstrate that available carrier conditions result in significant structural alterations of the proteins that they ship (Figure E). A negligible difference in structural protein changes was observed also when shipping temperatures were compared, whereby protein structures were different under cold and ambient conditions (Figure F). We also demonstrate that shipping methods, regardless of carrier, result in significant structural protein alterations. Overall, 1-day shipping had the highest impact on protein structure, followed by ground and then 2-3 day shipping (Figure G). Changes in protein melt curves were found to be $79.08 \%$ lower for proteins that were shipped via 2-3 day shipping as compared to 1-day (Figure G). Lastly, we demonstrate that plasma treated whey protein isolate experienced substantially lower structural alterations than their non-treated counterparts, reduced by $53.02 \%$ of structural attenuation (Figure $\mathrm{H}$ ).

We highlight here the importance of understanding the structural dynamics of protein macromolecules. In particular, this is essential with proteins that are used in food and nutrition sectors, such as commercially available whey protein powders. Due to their commercialization and wide availability, whey proteins are exposed to several environments that may have an impact on their structure and thus function. It is therefore essential to understand the structural alterations that are consequent to the conditions and environments these products are exposed to. Previous studies have investigated the effects of varying storage conditions on whey protein products, and alterations in surface structure and solubility were observed. ${ }^{11}$ Furthermore, storage time and temperature 
have both been found to affect protein solubility. ${ }^{12}$ Shipment and handling are physiochemical structure altering factors that have not been thoroughly investigated in light of protein structural changes, yet. Here we not only demonstrate that structural changes in commercially shipped protein products are evident among commercial shipping carriers, but also between the different shipping methods as well as ambient temperatures. More importantly, we demonstrate that PSM treated proteins do not experience the same extent of structural alterations, thereby suggesting that this method is effective in preserving protein resilience and thus structural integrity during environmental fluctuations. These implications could be of great benefit to both food and nutrition industries that rely on whey protein powder functionality and efficacy.

\section{Conclusions:}

Commercial carriers vary in shipping conditions and lead to an observed $9.14 \%$ variation in protein structure as measured by PTSA. We observed that the most rapid shipping option was not the most efficacious at preserving protein structure. The lowest structural alterations as measured by PTSA were observed to take place during the 2-3 day shipping option $(79.08 \%$ lower), followed by ground shipping. Furthermore, PSM treatment decreased the extent of protein structural changes by $53.02 \%$. These observations point to several potential findings. Of particular relevance are those that point to PSM as an effective preservation method of commercially available whey protein powders that are subjected to various shipping methods.

\section{Methods:}

A commercially available dry whey protein isolate powder was used for the study. Within that batch, treated powder was used as source material and untreated as control for the experiment. Source material was exposed to atmospheric plasma conditions using the Proprietary PSM technology (Ingredient Optimized TM, Plasma Nutrition, USA) technique described above. All non-control protein samples were shipped over an identical 462mile domestic journey within the United States. Overall, a total of 10 shipping and handling conditions were compared across three different shipping companies. Specifically, samples were shipped via three carriers, each of which utilized three shipping methods, namely 1-day, 2-3-day and ground shipping. Additionally, carrier 3 utilized a shipping method whereby sample was shipped under cold $\left(2-8^{\circ} \mathrm{C}\right)$ conditions.

Protein samples were diluted in phosphate buffer and Applied Biosystems Protein Thermal Shift dye was added according to the manufacturer's protocol. Each sample was run alongside three control samples to ensure accuracy (buffer only, buffer + control protein and buffer + control protein + Applied Biosystems Thermal Shift control ligand). Four replicates of each protein melt reaction were tested to ensure statistically significant results. All protein samples were stored at a constant temperature between $10 \mathrm{~F}$ and $20 \mathrm{~F}$ 
for the selected duration. The melt assay was run on a ThermoFisher Quantstudio realtime PCR system. Melt curve and melt peak data were obtained via Applied Biosystems Protein Thermal Shift software.

Statistical analysis was conducted via Prism 6 (GraphPad Software) using a one-way matched ANOVA $(p<0.05)$.

\section{Limitations:}

This study investigated the application of PSM on only one source of dry powdered whey protein isolate. While the whey protein isolate source material used in this investigation is commonly found in commercial use, there may be variations between the material used and other market-available whey protein isolate powders. Further studies are needed to examine the effect of PSM on a range of dry powder whey protein isolate sources.

\section{Funding Statement:}

This work was financially supported by Plasma Nutrition.

\section{Ethics Statement:}

All experiments were performed in vitro and did not involve the use of any living subject. No fraudulence is committed in performing these experiments or during processing of the data.

\section{Sources:}

1. Huynh, K. and Partch, C.L. (2015). Analysis of Protein Stability and Ligand Interactions by Thermal Shift Assay. Current Protocols in Protein Science, 79:28.9.1-28.9.14. DOI: 10.1002/0471140864.ps2809s79

2. Molina, D.M., Jafari, R., Ignatushchenko, M., Seki, T., Larsson, E.A., Dan, C., Sreekumar, L., Cao, Y. and Nordlund, P. (2013). Monitoring drug target engagement in cells and tissues using the cellular thermal shift assay. Science, 341(6141), pp.84-87. DOI: 10.1126/science.1233606

3. Kurganov, B.I. (2002). Kinetics of protein aggregation. Quantitative estimation of the chaperone-like activity in test-systems based on suppression of protein aggregation. Biochemistry (Moscow), 67(4), pp.409-422. DOI: 10.1023/A:1015277805345

4. Vedadi, M., Niesen, F.H., Allali-Hassani, A., Fedorov, O.Y., Finerty, P.J., Wasney, G.A., Yeung, R., Arrowsmith, C., Ball, L.J., Berglund, H. and Hui, R. (2006). Chemical screening methods to identify ligands that promote protein stability, protein crystallization, and structure determination. Proceedings of the National Academy of Sciences, 103(43), pp.15835-15840. DOI: 10.1073/pnas.0605224103

5. Chu, P.K., Chen, J.Y., Wang, L.P. and Huang, N. (2002). Plasma-surface modification of biomaterials. Materials Science and Engineering: $R$ : Reports, 36(5), pp.143-206. DOI: 10.1016/S0927-796X(02)00004-9 
6. Jeong, J.Y., Babayan, S.E., Tu, V.J., Park, J., Henins, I., Hicks, R.F. and Selwyn, G.S. (1998). Etching materials with an atmospheric-pressure plasma jet. Plasma Sources Science and Technology, 7(3), p.282. DOI: 10.1088/0963-0252/7/3/005

7. Shenton, M.J. and Stevens, G.C. (2001). Surface modification of polymer surfaces: atmospheric plasma versus vacuum plasma treatments. Journal of Physics D: Applied Physics, 34(18), p.2761. DOI: 10.1088/0022-3727/34/18/308

8. Kinsella, J.E. and Melachouris, N. (1976). Functional properties of proteins in foods: a survey. Critical Reviews in Food Science \& Nutrition, 7(3), pp.219-280. DOI: $10.1080 / 10408397609527208$

9. McIntosh, G.H., Royle, P.J., Le Leu, R.K., Regester, G.O., Johnson, M.A., Grinsted, R.L., Kenward, R.S. and Smithers, G.W. (1998). Whey proteins as functional food ingredients? International Dairy Journal, 8(5-6), pp.425-434. DOI: 10.1016/S0958-6946(98)00065-X

10. Regester, G.O., McIntosh, G.H., Lee, V.W.K. and Smithers, G.W. (1996). Whey proteins as nutritional and functional food ingredients. Food Australia, 48(3), pp.123-127.

11. Fyfe, K.N., Kravchuk, O., Le, T., Deeth, H.C., Nguyen, A.V. and Bhandari, B. (2011). Storage induced changes to high protein powders: influence on surface properties and solubility. Journal of the Science of Food and Agriculture, 91(14), pp.2566-2575. DOI: 10.1002/jsfa.4461

12. Anema, S.G., Pinder, D.N., Hunter, R.J. and Hemar, Y. (2006). Effects of storage temperature on the solubility of milk protein concentrate (MPC85). Food hydrocolloids, 20(2), pp.386-393. DOI: 10.1016/j.foodhyd.2005.03.015 